\title{
Differences in chronic conditions and lifestyle behaviour between people with a history of cancer and matched controls
}

\section{Narelle M Berry BSc, BAppSci(Hons), PhD Postdoctoral Research Fellow 1,2}

Michelle D Miller BSc, MNutDiet, PhD Associate Professor and Head of Discipline of Nutrition and Dietetics ${ }^{1}$

Richard JWoodman BSc, MBiostats, PhD Associate Professo in Epidemiology and Biostatistics. Flinders Centre for Epidemiology and Biostatistics

John Coveney PhD, MHPEd, BSc(Hons) Dean, School of Health Sciences ${ }^{1}$

James Dollman BSc, MSc, PhD Associate Professor

Catherine R Mackenzie PhD, BA(Hons), RN Associate Lecturer Southgate Institute fo Health, Society and Equity

Bogda Koczwara BMBS, FRACP

Medical Oncologist,

Flinders Centre for

Innovation in Cancer

1Faculty of Medicine, Nursing and Health Sciences,

Flinders University, Adelaide, SA

2 School of Health Sciences, University of South Australia,

Adelaide, SA.

narelle.berry@ flinders.edu.au

MJA 2014; 201: 96-100 doi: 10.5694/mjal3.1070

Online first 14/07/14
T ealth care of cancer survivors has traditionally focused on detecting cancer recurrence, with less focus on managing chronic diseases. This is despite growing evidence that cancer survivors have a significant burden of non-cancer chronic illness. ${ }^{1,2}$ Most of this evidence has come from population-based studies, ${ }^{3,4}$ with few case-control studies. ${ }^{5}$ An Australian population-based study showed that long-term cancer survivors were more likely than controls to report multiple long-term conditions. ${ }^{6}$ Another study found that people with a history of cancer were nearly $50 \%$ more likely to die of non-cancer causes than the general population.7

Very few studies have looked at the impact of sex on the association between cancer and comorbidity. A Dutch cancer registry study of colon cancer patients showed that comorbidity was more likely in men and in patients with lower socioeconomic status (SES), 8,9 consistent with general patterns of comorbidity in people without cancer. ${ }^{10}$ Another study of cancer patients from the Dutch registry also showed a higher likelihood of comorbidity in men and a $50 \%$ greater risk of serious comorbidity in those with low SES.11

Our aim was to determine whether individuals previously diagnosed with cancer reported a higher prevalence of chronic conditions or different lifestyle behaviour than age- and sexmatched controls. We also examined self-reported health in those with cancer and chronic conditions compared with those with cancer and no chronic conditions.

\section{Methods}

Data were collected using the South Australian Monitoring and Surveillance System (SAMSS) from 1 January 2010 to 31 March 2012.

Abstract

Objective: To determine whether people with a history of cancer have a higher prevalence of chronic conditions or different lifestyle behaviour compared with controls.

Design, setting and participants: Cross-sectional, self-reported data from a telephone survey conducted between 1 January 2010 and 31 March 2012 of adult residents of South Australia who self-reported a previous cancer diagnosis (cases) and randomly selected age- and sex-matched residents with no cancer diagnosis (controls).

Main outcome measures: Self-reported medically diagnosed cardiovascular disease, hypertension, hyperlipidaemia, diabetes and osteoporosis; lifestyle behaviour (smoking, physical activity and diet); body mass index (BMI); psychological distress and self-reported health.

Results: A total of 2103 cases and 4185 controls were included in the analyses. For men, after adjusting for age, cancer survivors were more likely than controls to have ever had cardiovascular disease $(P<0.001)$, high blood pressure $(P=0.001)$, high cholesterol $(P<0.001)$ and diabetes $(P=0.04)$. These associations remained significant after controlling for socioeconomic status (SES), with the exception of high blood pressure $(P=0.09)$. For women, there was an increased prevalence of high cholesterol $(P=0.005)$, diabetes $(P=0.02)$ and osteoporosis $(P=0.005)$ in cancer cases, but after adjusting for SES, these associations were no longer significant. Women with a previous cancer diagnosis were more likely than controls to have ever smoked, after adjusting for SES $(P=0.001)$. There were no other differences in lifestyle behaviour or BMI between cases and controls for men or women.

Conclusion: Despite similar lifestyle habits and BMI, the prevalence of chronic conditions was significantly higher among people with a history of cancer than among controls without cancer. This supports the importance of chronic disease management as part of health care after a diagnosis of cancer.

SAMSS is a computer-assisted telephone interview survey that monitors self-reported trends in diseases, health problems, risk factors and health service use over time. ${ }^{12}$

This study was approved by the human research ethics committees at the South Australian Department for Health and Ageing and Flinders University, and participants gave verbal informed consent before participating.

Questions relating to cancer diagnosis for participants aged 18 years or over were used to identify those with a previous cancer diagnosis and up to two age- and sex-matched controls per cancer case. We used propensity score matching techniques ${ }^{13}$ to probabilistically match about two survey respondents without cancer to each survey respondent with current cancer or a history of cancer. Matching was performed according to 5-year age groups (from 15-20 years to 95-100 years) and sex.

\section{Data items}

The questions used are shown in Appendix 1 (online at mja.com.au). Prevalence of chronic conditions was assessed by asking respondents if they had ever been medically diagnosed with cardiovascular disease, high blood pressure, high cholesterol, diabetes and osteoporosis. Selfreported health was determined by respondents rating their health on a five-category scale from excellent to poor. Psychological distress was assessed using the 10-item Kessler 
Psychological Distress Scale (K10).14 Self-reported height and bodyweight were used to calculate body mass in$\operatorname{dex}$ (BMI).

Physical activity was measured using six questions from the Active Australia Survey. ${ }^{15}$ Answers to these questions were summed into three categories to establish whether sufficient physical activity was being performed, in accordance with national guidelines. ${ }^{16}$

SES was assessed using the SocioEconomic Indexes for Areas (SEIFA) score. ${ }^{17}$ Data were also collected on education, social environment, work status, family structure, income and first language other than English.

\section{Statistical analysis}

Demographic variables were compared using $\chi^{2}$ tests. We used conditional logistic regression to assess the association between cancer status and each of the chronic condition prevalence outcomes and whether participants met fruit and vegetable intake recommendations. We used a random-effects ordinal logistic regression model to assess the association between cancer status and self-rated health and sufficiency of exercise. For this, the matched group of participants (ie, one case and two controls) was the random effect. For each analysis, we first adjusted for age. Then, to control for any differences between groups in SES, we adjusted for SEIFA score, with education, social environment, work status, family structure, income and first language other than English also entered into the model as potential confounders. All analyses were stratified by sex.

As a sensitivity analysis, we estimated morbidity effects for cancer cases diagnosed within the previous 5 years compared with those diagnosed more than 5 years previously.

The data were not survey weighted as we used only a subset of the original survey data for this study. All analyses were performed using Stata, version 12.1 (StataCorp). Each analysis was performed as a two-sided hypothesis test with variables considered significant if $P<0.05$.

\section{Results}

From 1 January 2010 to 31 March 2012, 40528 dwellings in South Australia were selected. Of these, 9711 were out of scope (not eligible or disconnected phone numbers), 5869 residents refused to participate, 2938 could not be contacted, 966 were non-English speakers, and 1536 were unavailable or incapacitated. In total, 19508 interviews were completed, achieving a response rate of $63 \%$ of those in scope. Of these, 2103 respondents were identified as having been diagnosed with cancer (cases) and were age- and sex-matched to 4185 respondents without previous cancer (controls). Although we were able to match $96.3 \%$ (2025/2103) of cancer respondents to two controls, suitable matching could not be done for some older respondents ( $\geqslant 90$ years of age): 18 cases were unmatched, and 30 were matched with only one control. All matched and unmatched cases were included in the analysis.

In the cancer group, 938 participants (44.6\%) were men and 1165 (55.4\%) were women; the mean (SD) age was 69.6 (11.6) years for men and 68.0 (12.5) years for women. In the control group, 1860 participants $(44.4 \%)$ were men and 2325 (55.6\%) were women; the mean (SD) age was 69.3 (11.6) years for men and 67.8 (12.5) years for women. There were no significant differences in mean ages between the groups.

The demographic profile of the sample is outlined in Appendix 2 (online at mja.com.au). Compared with controls, a greater proportion of women in the cancer group reported high to very high psychological distress, and greater proportions of both men and women in the cancer group reported poor self-rated health. There were no significant differences between cases and controls for BMI.

Skin cancer (melanoma and nonmelanoma) was the most commonly reported cancer $(35.7 \%)$, followed by breast cancer, male (prostate and testicular) cancer, and gastrointestinal cancer (Appendix 3, online at mja. com.au).

Mean (SD) time since diagnosis for the cancer group was 10.86 (10.38) years, with 5.5\% (114/2063) diagnosed less than 1 year before the survey, 28.0\% (578/2063) diagnosed $1-4.99$ years previously, and $66.5 \%$ (1371/2063) diagnosed 5 or more years previously.

For men, when adjusted for age, cancer survivors were significantly more likely than controls to have ever had cardiovascular disease, high blood pressure, high cholesterol and diabetes (Box 1). When adjusted for age and SES, the increased odds remained for all variables except high blood pressure. For women, when adjusted for age, cancer survivors were significantly more likely than controls to have ever had high cholesterol, diabetes and osteoporosis. However, after adjusting for SES, these associations were no longer significant.

In the sensitivity analysis comparing cancer cases diagnosed within the previous 5 years with those diagnosed more than 5 years previously, we found no substantive differences in the results except for the prevalence of hypertension among men diagnosed with cancer more than 5 years ago (significantly increased odds for cancer cases compared with controls in the adjusted model: odds ratio, 1.36; $95 \%$ CI, 1.03-1.80; $P=0.03$ ).

For men, there were no significant differences between cancer cases and controls for any lifestyle variables (Box 2). For women, there was a significant association between cancer diagnosis and smoking status, which remained significant after being fully adjusted. There was also a significant association with physical activity status in women, which was no longer significant after adjusting for SES.

There was a significant association between self-reported health and the presence of one or more chronic conditions among cancer cases. For men, 35.9\% (265/739) of those with cancer and one or more chronic conditions rated their health as fair or poor compared with $19.6 \%(39 / 199)$ of those with cancer alone $(P<0.001)$. For women, these figures were $38.9 \%$ (350/900) compared with $19.2 \%$ $(51 / 265)(P<0.001)$. A similar pattern was seen in the control group, with $26.8 \%$ (341/1272) of men with one or more chronic conditions reporting fair or poor health compared with $12.4 \%$ (73/588) of men with no chronic conditions $(P<0.001)$, and $27.4 \%(462 / 1686)$ compared with $12.8 \%(82 / 639)$ of women $(P<0.001)$.

\section{Discussion}

We found that individuals with a history of cancer had a higher prevalence of chronic conditions, compared with 
age- and sex-matched controls. Men with a history of cancer had increased odds of ever having had cardiovascular disease, high blood pressure, high cholesterol and diabetes, which remained significant after adjusting for SES. Among women, the apparent relationship between cancer status and self-reported high cholesterol, diabetes and osteoporosis was largely explained by SES, with the association weakening after adjusting for SES factors. These results support previous research findings that cancer survivors are more likely to report having other long-term comorbidities, 5,6 with men more at risk than women. ${ }^{8}$

We observed these differences in chronic disease prevalence between cancer survivors and controls despite similar lifestyle behaviour between the groups, including diet and physical activity, and similar levels of overweight or obesity. These findings are similar to those of another Australian study, which showed that women with a history of cancer were more likely to be smokers but there were no differences in physical activity or fruit and vegetable consumption between cancer patients and controls. ${ }^{18}$ Such observations support an association between cancer and chronic disease, although the underlying mechanisms and causal direction are unknown.

It is possible that chronic illness itself may predispose to cancer. Increased risk of cancer has been reported in patients with diabetes, ${ }^{19}$ and cardiovascular disease and osteoporosis share the common aetiological factors of inactivity and obesity with cancer. Likewise,

\section{Odds ratios (ORs) for self-reported chronic conditions among men and women, for cancer cases versus matched controls}

\begin{tabular}{|c|c|c|c|c|c|c|}
\hline Chronic condition* & Controls & Cancer cases & Age-adjusted OR $(95 \% \mathrm{Cl})$ & $P$ & Adjusted $\mathrm{OR}^{\dagger}(95 \% \mathrm{Cl})$ & $P$ \\
\hline Men & $n=1860$ & $n=938$ & & & & \\
\hline \multicolumn{7}{|c|}{ Cardiovascular disease } \\
\hline Yes & $443(23.8 \%)$ & 281 (30.0\%) & 1.39 (1.16-1.67) & $<0.001$ & $1.48(1.15-1.90)$ & 0.002 \\
\hline No & $1417(76.2 \%)$ & $657(70.0 \%)$ & 1.00 & & 1.00 & \\
\hline \multicolumn{7}{|l|}{ High blood pressure } \\
\hline Yes & 901 (48.4\%) & 515 (54.9\%) & $1.30(1.11-1.53)$ & 0.001 & $1.19(0.97-1.47)$ & 0.09 \\
\hline No/don't know & 959 (51.6\%) & $423(45.1 \%)$ & 1.00 & & 1.00 & \\
\hline \multicolumn{7}{|l|}{ High cholesterol } \\
\hline Yes & $752(40.4 \%)$ & 448 (47.8\%) & 1.35 (1.15-1.59) & $<0.001$ & $1.43(1.16-1.76)$ & 0.001 \\
\hline No/don't know & 1108 (59.6\%) & $490(52.2 \%)$ & 1.00 & & 1.00 & \\
\hline \multicolumn{7}{|l|}{ Diabetes } \\
\hline Yes & 300 (16.1\%) & $180(19.2 \%)$ & $1.24(1.01-1.52)$ & 0.04 & $1.47(1.11-1.94)$ & 0.007 \\
\hline No/don't know & 1554 (83.6\%) & 756 (80.6\%) & & & & \\
\hline Missing & $6(0.3 \%)$ & $2(0.2 \%)$ & & & & \\
\hline \multicolumn{7}{|l|}{ Osteoporosis } \\
\hline Yes & $85(4.6 \%)$ & $50(5.3 \%)$ & $1.18(0.82-1.70)$ & 0.38 & 1.11 (0.67-1.85) & 0.69 \\
\hline No & 1763 (94.8\%) & 879 (93.7\%) & & & & \\
\hline Missing & $12(0.6 \%)$ & $9(1.0 \%)$ & & & & \\
\hline Women & $n=2325$ & $n=1165$ & & & & \\
\hline \multicolumn{7}{|c|}{ Cardiovascular disease } \\
\hline Yes & $328(14.1 \%)$ & $183(15.7 \%)$ & 1.11 (0.91-1.37) & 0.30 & $1.17(0.86-1.58)$ & 0.31 \\
\hline No & 1997 (85.9\%) & $982(84.3 \%)$ & 1.00 & & 1.00 & \\
\hline \multicolumn{7}{|l|}{ High blood pressure } \\
\hline Yes & 1192 (51.3\%) & 605 (51.9\%) & $1.02(0.88-1.19)$ & 0.76 & $0.94(0.77-1.16)$ & 0.58 \\
\hline No/don't know & $1133(48.7 \%)$ & 560 (48.1\%) & 1.00 & & 1.00 & \\
\hline \multicolumn{7}{|l|}{ High cholesterol } \\
\hline Yes & $997(42.9 \%)$ & 557 (47.8\%) & $1.23(1.07-1.43)$ & 0.005 & $1.04(0.85-1.30)$ & 0.67 \\
\hline No/don't know & 1328 (57.1\%) & 608 (52.2\%) & 1.00 & & 1.00 & \\
\hline \multicolumn{7}{|l|}{ Diabetes } \\
\hline Yes & $260(11.2 \%)$ & $164(14.1 \%)$ & $1.28(1.04-1.58)$ & 0.02 & $1.19(0.88-1.62)$ & 0.26 \\
\hline No & 2062 (88.7\%) & 998 (85.7\%) & & & & \\
\hline Missing & $3(0.1 \%)$ & $3(0.2 \%)$ & & & & \\
\hline \multicolumn{7}{|l|}{ Osteoporosis } \\
\hline Yes & 359 (15.4\%) & $226(19.4 \%)$ & $1.31(1.08-1.58)$ & 0.005 & $1.24(0.94-1.65)$ & 0.12 \\
\hline No & 1942 (83.5\%) & 930 (79.8\%) & & & & \\
\hline Missing & $24(1.0 \%)$ & $9(0.8 \%)$ & & & & \\
\hline
\end{tabular}

* Respondents were asked if they had ever been medically diagnosed with each condition. $†$ Adjusted for age, Socio-Economic Indexes for Areas score, education, social environment, work status, family structure, income and first language other than English. ORs were obtained using conditional logistic regression. 
cancer or cancer treatment may predispose to chronic disease. Endothelial dysfunction, platelet activation and upregulation of prothrombotic factors as a result of anticancer therapy have been postulated, but other mechanisms, yet to be defined, could be involved. ${ }^{20}$ Similar associations between therapy and development of chronic disease have been observed in the treatment of HIV, where chronic inflammation and disruption of normal immunity have been proposed as an aetiological mechanism. ${ }^{21}$
Our findings indicate that men with cancer are more likely to report chronic illness, consistent with other studies. ${ }^{8}$ This may be due to different types of cancers observed in men, different cancer treatments used or a general greater predisposition to chronic illness in men. ${ }^{10}$ It is possible that the association is due to men being more likely to have undiagnosed chronic disease because they have fewer interactions with health and medical services. As diagnosis and treatment of cancer brings men under the surveillance of health and medical services, where other chronic conditions are more likely to be identified, the difference between male cancer survivors and controls may be a result of underdiagnosis of chronic conditions in the controls.

For women, the association of cancer with chronic illness was no longer significant after adjusting for SES. It is unclear why the association between cancer and chronic illness differs between men and women, and may reflect differences in cancer type, health

\section{Odds ratios (ORs) for lifestyle behaviour among men and women, for cancer cases versus matched controls}

\begin{tabular}{|c|c|c|c|c|c|c|}
\hline Lifestyle behaviour & Controls & Cancer cases & Age-adjusted OR (95\% Cl) & $P$ & Adjusted OR* $(95 \% \mathrm{Cl})$ & $P$ \\
\hline Men & $n=1860$ & $n=938$ & & & & \\
\hline \multicolumn{7}{|l|}{ Ever smoked } \\
\hline Yes & $1282(68.9 \%)$ & $675(72.0 \%)$ & $1.16(0.97-1.38)$ & 0.10 & $1.05(0.84-1.32)$ & 0.65 \\
\hline No & $577(31.0 \%)$ & $263(28.0 \%)$ & & & & \\
\hline Missing & $1(0.1 \%)$ & 0 & & & & \\
\hline \multicolumn{7}{|l|}{ Physical activity } \\
\hline Sufficient & $817(43.9 \%)$ & $412(43.9 \%)$ & $1.01(0.87-1.17)$ & 0.93 & $0.95(0.79-1.13)$ & 0.55 \\
\hline Active but not sufficient & $537(28.9 \%)$ & $269(28.7 \%)$ & & & & \\
\hline Not active & $461(24.8 \%)$ & $234(24.9 \%)$ & & & & \\
\hline Missing & $45(2.4 \%)$ & $23(2.5 \%)$ & & & & \\
\hline \multicolumn{7}{|c|}{ Recommended fruit intake ( 2 servings/day) } \\
\hline Yes & $780(41.9 \%)$ & $410(43.7 \%)$ & $1.08(0.92-1.27)$ & 0.35 & $1.14(0.95-1.38)$ & 0.16 \\
\hline No & $1074(57.7 \%)$ & $524(55.9 \%)$ & 1.00 & & 1.00 & \\
\hline Missing & $6(0.3 \%)$ & $4(0.4 \%)$ & & & & \\
\hline \multicolumn{7}{|c|}{ Recommended vegetable intake ( 5 servings/day) } \\
\hline Yes & $195(10.5 \%)$ & $112(11.9 \%)$ & $1.16(0.91-1.49)$ & 0.24 & $1.11(0.83-1.50)$ & 0.47 \\
\hline No & $1631(87.7 \%)$ & $808(86.1 \%)$ & 1.00 & & 1.00 & \\
\hline Missing & $34(1.8 \%)$ & $18(1.9 \%)$ & & & & \\
\hline Women & $n=2325$ & $n=1165$ & & & & \\
\hline \multicolumn{7}{|l|}{ Ever smoked } \\
\hline Yes & $1071(46.1 \%)$ & $589(50.6 \%)$ & $1.19(1.03-1.37)$ & 0.015 & $1.42(1.17-1.73)$ & 0.001 \\
\hline No & $1253(53.9 \%)$ & $576(49.4 \%)$ & & & & \\
\hline Missing & $1(0.04 \%)$ & 0 & & & & \\
\hline \multicolumn{7}{|l|}{ Physical activity } \\
\hline Sufficient & $886(38.1 \%)$ & $409(35.1 \%)$ & $1.19(1.04-1.36)$ & 0.01 & $1.16(0.99-1.37)$ & 0.07 \\
\hline Active but not sufficient & $756(32.5 \%)$ & $368(31.6 \%)$ & & & & \\
\hline Not active & $629(27.1 \%)$ & $364(31.2 \%)$ & & & & \\
\hline Missing & $54(2.3 \%)$ & $24(2.1 \%)$ & & & & \\
\hline \multicolumn{7}{|c|}{ Recommended fruit intake ( 2 servings/day) } \\
\hline Yes & $1284(55.2 \%)$ & $670(57.5 \%)$ & $1.10(0.95-1.27)$ & 0.20 & $1.10(0.91-1.31)$ & 0.99 \\
\hline No & $1038(44.7 \%)$ & $494(42.4 \%)$ & 1.00 & & 1.00 & \\
\hline Missing & $3(0.1 \%)$ & $1(0.1 \%)$ & & & & \\
\hline \multicolumn{7}{|c|}{ Recommended vegetable intake ( 5 servings/day) } \\
\hline Yes & $326(14.0 \%)$ & $167(14.3 \%)$ & $1.03(0.84-1.26)$ & 0.76 & $1.18(0.92-1.51)$ & 0.19 \\
\hline No & $1985(85.4 \%)$ & $986(84.6 \%)$ & 1.00 & & 1.00 & \\
\hline Missing & $14(0.6 \%)$ & $12(1.0 \%)$ & & & & \\
\hline
\end{tabular}

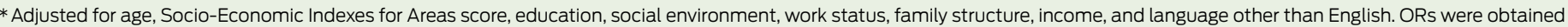
using conditional logistic regression for fruit and vegetable intake, and random-effects ordinal logistic regression for smoking status and physical activity. 
utilisation or other factors that warrant further study. Our observation underscores the importance of SES in the overall disease burden after a diagnosis of cancer, especially for women. Low SES has been associated with worse cancer outcomes ${ }^{22}$ and with higher rates of chronic disease. ${ }^{23}$

Our study indicates that those with a history of cancer face an additional burden of chronic illness, which may contribute to increased distress and a perception of poor health. In our study, those with a cancer diagnosis and at least one additional chronic condition were more likely to report poor health compared with those with cancer alone. While a similar association was found for controls with a chronic condition, these findings highlight the importance of managing chronic illness after a cancer diagnosis. Current models of care may not give the same attention to chronic disease management, despite its potential impact on health care use, costs and productivity. ${ }^{24}$ Novel approaches to chronic disease management incorporating self-management strategies have been shown to be efficacious and cost-effective in the non-cancer setting, ${ }^{25}$ and their use for patients with a cancer diagnosis should be explored.

Our study was unable to provide detailed information on how many respondents with previous cancer were treated with curative intent, but the data indicate that most (94.5\%) had received a cancer diagnosis more than a year prior, with two-thirds diagnosed more than 5 years previously. Of note, $35.7 \%$ of the cancer patients had skin cancer, where information about the histological type and type of cancer treatment was not available. To examine any impact of the high proportion of skin cancers on our findings, we repeated the analysis both with and without skin cancer cases and found no difference in the odds of chronic conditions.

Our study offers insight into the disease burden after cancer diagnosis that warrants further investigation. Its limitations relate to the self-reported nature of the data, the cross-sectional study design, and the potential for selection bias of participants. As only residents who had access to a telephone and were well enough to answer it were able to take part, this may select against residents with or without cancer who were very ill or hospitalised. This is in addition to the survival bias inherent in prevalence studies, as those who had more severe cancer or more severe comorbidities may have died before they could participate. However, this potential bias would favour selection of long-term cancer survivors where the information on chronic illness is more relevant.

Research is needed into chronic illness after cancer diagnosis, with a focus on prevalence, underlying mechanisms and development of optimal management strategies.

Competing interests: No relevant disclosures.

\section{Received 29 May 2013, accepted 22 May 2014}

1 Janssen-Heijnen ML, Schipper RM, Razenberg PP, et al. Prevalence of co-morbidity in lung cancer patients and its relationship with treatment: a population-based study. Lung Cancer 1998; 21: 105-113.

2 Jørgensen TL, Hallas J, Friis S, Herrstedt J. Comorbidity in elderly cancer patients in relation to overall and cancer-specific mortality. Br J Cancer 2012; 106: 1353-1360.

3 Elliott J, Fallows A, Staetsky L, et al. The health and well-being of cancer survivors in the UK: findings from a population-based survey. $\mathrm{Br} J$ Cancer 2011; 105 Suppl 1: S11-S20.

4 Sarfati D, Tan L, Blakely T, Pearce N. Comorbidity among patients with colon cancer in New Zealand. N Z Med J 2011; 124: 76-88.

5 Yabroff KR, Lawrence WF, Clauser S, et al. Burden of illness in cancer survivors: findings from a population-based national sample. J Natl Cancer Inst 2004; 96: 1322-1330.

6 Eakin EG, Youlden DR, Baade PD, et al. Health status of long-term cancer survivors: results from an Australian population-based sample. Cancer Epidemiol Biomarkers Prev 2006; 15: 1969-1976.

7 Baade PD, Fritschi L, Eakin EG. Non-cancer mortality among people diagnosed with cancer (Australia). Cancer Causes Control 2006; 17: 287-297.

8 Coebergh J, Janssen-Heijnen M, Post PN, Razenberg PP. Serious co-morbidity among unselected cancer patients newly diagnosed in the southeastern part of The Netherlands in 1993-1996. J Clin Epidemiol 1999; 52: 1131-1136.

9 Van Leersum NJ, Janssen-Heijnen ML, Wouters $\mathrm{MW}$, et al. Increasing prevalence of comorbidity in patients with colorectal cancer in the South of the Netherlands 1995-2010. Int J Cancer 2013; 132: 2157-2163.

10 Schäfer I, Hansen H, Schön G, et al. The influence of age, gender and socio-economic status on multimorbidity patterns in primary care. First results from the multicare cohort study. BMC Health Serv Res 2012; 12: 89.

11 Louwman WJ, Aarts MJ, Houterman S, et al. A 50\% higher prevalence of life-shortening chronic conditions among cancer patients with low socioeconomic status. Br J Cancer 2010; 103: 1742-1748.

12 SA Health. South Australian Monitoring and Surveillance System (SAMSS). Survey methodology. SAMSS Technical Paper Series No. 1/04. Adelaide: Government of South Australia, 2004. https://health.adelaide.edu. au/pros/docs/reports/report_samss_tech_ paper.pdf (accessed May 2014).

13 Leuven E, Sianesi B. PSMATCH2: Stata module to perform full Mahalanobis and propensity score matching, common support graphing, and covariate imbalance testing. Boston: Statistical Software Components, Boston College Department of Economics, 2003.

14 Kessler RC, Andrews G, Colpe LJ, et al. Short screening scales to monitor population prevalences and trends in non-specific psychological distress. Psychol Med 2002; 32: 959-976.

15 Australian Institute of Health and Welfare. The Active Australia Survey: a guide and manual for implementation, analysis and reporting. Canberra: AlHW, 2003. (AlHW Cat. No. CVD 22.)

16 Department of Health and Ageing. An active way to better health. National physical activity guidelines for adults. Canberra: Commonwealth of Australia, 1999.

17 Australian Bureau of Statistics. SocioEconomic Indexes for Areas (SEIFA). Technical paper. Canberra: ABS, 2011. (ABS Cat. No. 2033.0.55.001.)

18 Eakin EG, Youlden DR, Baade PD, et al. Health behaviors of cancer survivors: data from an Australian population-based survey. Cancer Causes Control 2007; 18: 881-894.

19 Giovannucci E, Harlan DM, Archer MC, et al. Diabetes and cancer: a consensus report. $C A$ Cancer J Clin 2010; 60: 207-221.

20 Soultati A, Mountzios G, Avgerinou C, et al. Endothelial vascular toxicity from chemotherapeutic agents: preclinical evidence and clinical implications. Cancer Treat Rev 2012; 38: 473-483.

21 Deeks SG. HIV infection, inflammation, immunosenescence, and aging. Annu Rev Med 2011; 62: 141-155.

22 Byers TE, Wolf HJ, Baver KR, et al. The impact of socioeconomic status on survival after cancer in the United States: findings from the National Program of Cancer Registries Patterns of Care Study. Cancer 2008; 113: 582-591.

23 Lawrence PS. Chronic illness and socioeconomic status. Public Health Rep 1948; 63: 1507-1521.

24 Tang DH, Alberts DS, Nevins R, et al. Health care expenditures, hospitalizations, and productivity associated with cancer in US employer settings. J Occup Environ Med 2012; 54: 1453-1460.

25 Battersby M, Von Korff M, Schaefer J, et al. Twelve evidence-based principles for implementing self-management support in primary care. Jt Comm J Qual Patient Saf 2010; 36: 561-570. 